ORIGINAL PAPER

\title{
FUNCTIONAL MR UROGRAPHY - PRINCIPLES AND APPLICATIONS IN CONGENITAL OBSTRUCTIVE UROPATHIES
}

\author{
R.G. Nicolaescu ${ }^{1,2}$, R. Capșa ${ }^{2,3}$, R.C. Datu ${ }^{1,2}$, T. Enache ${ }^{1}$, Ioana Gabriela Lupescu ${ }^{2,3}$, I. Gherghina ${ }^{2,4}$ \\ 1"Grigore Alexandrescu" Emergency Children's Hospital \\ ${ }^{2}$ Carol Davila University of Medicine and Pharmacy of Bucharest, Romania \\ ${ }^{3}$ Fundeni Clinical Institute \\ 4"'Alessandrescu-Rusescu" Mother and Child's Health Institute
}

Corresponding author: Tudor Enache

Email: tudor.d.enache@gmail.com

\begin{abstract}
Congenital obstructive Uropathies are a leading cause of Chronic Kidney Disease in children. The gold-standard for renal functional imaging is Diuretic Renal Scintigraphy. Different alternatives to Renal Scintigraphy have been suggested, many of them based on Magnetic Resonance Imaging (MRI). The accurate morphologic details of MRI with its more specific application - MR-Urography are well known. The prospect of adding functional information is very attractive, as it will make the MRU a comprehensive "all in one" examination for obstructive uropathies. We chose to investigate DCE MR urography and DTI as tools for the evaluation of congenital obstructive uropathies. Also, we suggest a different technique that describes the urinary flow in the collecting system - phase contrast imaging of the urinary flow in the renal pelvis. Therefore we performed three different studies, all with different sets of patients.
\end{abstract}

KEYWORDS: $m$ r urography, congenital obstructive uropathies

\section{INTRODUCTION}

Congenital obstructive Uropathies are a leading cause of Chronic Kidney Disease in children [1]. The hallmark of these diseases is the dilation of the urinary tract - the hydronephrosis. Given recent advances in imaging and an increase of prenatal ultrasound screening, the number of fetuses and children with urinary tract dilation increased tremendously. Some epidemiologic studies report a prevalence of congenital hydronephrosis up to 11.5 cases in 10,000 living newborns [2], while antenatal studies identified antenatal hydronephrosis in up to $1 \%$ of pregnancies [3]. However, in most cases, the dilatation regresses, or persists but without significant functional renal damage, a meta- analysis study reporting only $36 \%$ of cases requiring corrective treatment [3].

Current diagnostic protocols and guidelines are based firstly, on ultrasound [4], as shown in Figure 1. For low degrees of dilation, the risk of renal damage is low, therefore only follow-up is recommended. However, for patients with high grade hydronephrosis, some sort of functional imaging is required to detect those patients with altered renal function that need corrective treatment. The gold-standard for renal functional imaging is Diuretic Renal Scintigraphy. This technique uses a radioactive tracer (99mTc-MAG3 or 99mTc-DTPA) and by measuring its uptake and excretion it can calculate the Split Renal Function for each kidney, a parameter used to guide treatment [5]. However this technique is not readily available, 


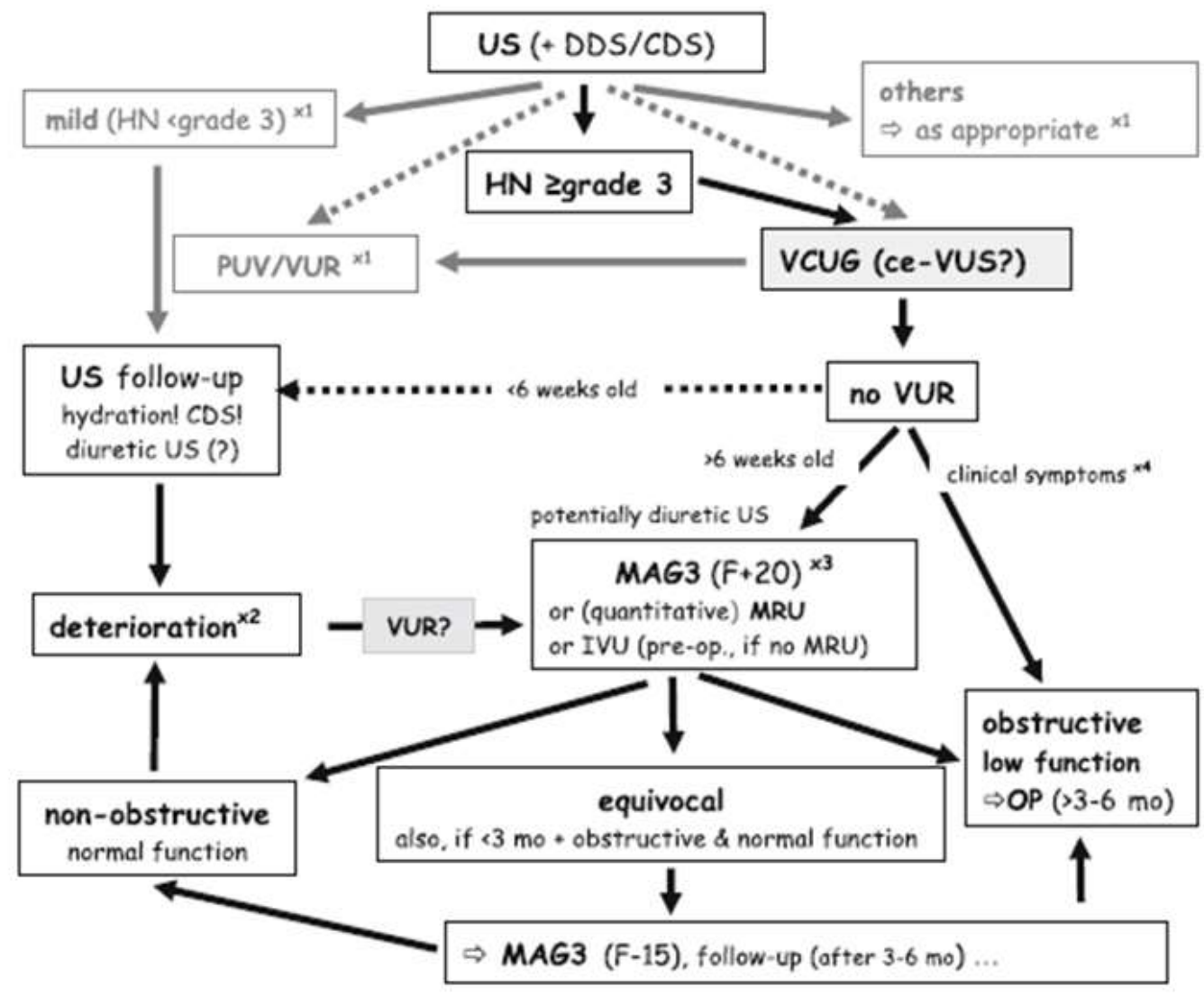

Figure 1 - Imaging protocol for Urinary tract dilation in children. From Riccabona M, Avni FE, Blickman JG, Dacher J-N, Darge K, Lobo ML, et al. Imaging recommendations in pediatric uroradiology. Pediatr Radiol [Internet] 2009;39(8):891-8.

Different alternatives to Renal the urinary flow in the renal pelvis. Therefore Scintigraphy have been suggested, many of them based on Magnetic Resonance Imaging (MRI). The accurate morphologic details of MRI with its more specific application - MRUrography are well known. The prospect of adding functional information is very attractive, as it will make the MRU a comprehensive "all in one" examination for obstructive uropathies.

Different techniques of functional MRU (fMRU) have been developed, which investigate different aspects of the renal function: Dynamic Contrast Enhanced MR Urography [6-12], Diffusion Tensor Imaging [13-19], Arterial Spin Labeling non-contrast perfusion imaging $[20,21]$ or BOLD imaging $[22,23]$. We chose to investigate DCE MR urography and DTI as tools for the evaluation of congenital obstructive uropathies. Also, we suggest a different technique that describes the urinary flow in the collecting system - phase contrast imaging of we performed three different studies, all with different sets of patients.

\section{MATERIALS AND METHODS}

\section{DCE MR Urography}

This technique involves imaging the kidneys dynamically during contrast medium administration for about 10-15 minutes. The signal characteristics over time are used to create a plot - the signal intensity-time curve. By analyzing the shape and amplitude of the curve, information about the renal function is extracted, most importantly, the split renal function (SRF).

Patient preparation is important. Good hydration is mandatory. Furthermore, diuretic load by Furosemide IV administration is helpful reducing artifacts and to make the method more sensitive. 
We imaged one patient with obstructed pyelo-ureteral junction on a 1.5Tesla machine (Magnetom Aera, Siemens@), Erlangen, Germany). A 3D T1 MR Angiography sequence was used to acquire the images. The sequence should be very fast, with temporal resolution of less than 10 seconds. Specifically, we used a TWIST sequence (Time-resolved angiography With Interleaved Stochastic Trajectories) which allows scanning times as fast as 2.5 seconds. The field of view is set in coronal plane and must include the kidneys, bladder and aorta. The scanning is started after injection of the contrast medium and is repeated continuously for 10-15 minutes (Figure 2).

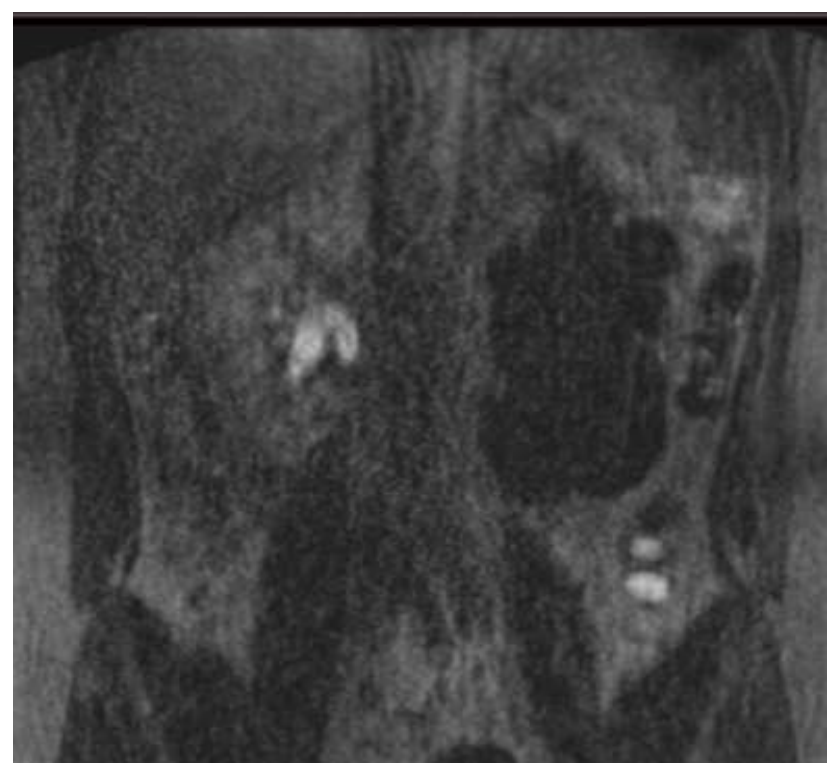

Figure 2 - Snapshot of excretion phase in the dynamic scan of the kidneys by DCE Note the severely dilated, hypo functioning left kidney (Personal collection).

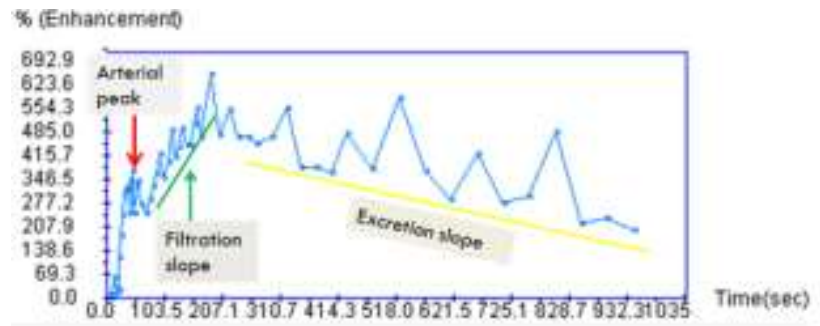

Figure 3 - Signal intensity - time curve with its components.

The images are post-processed with dedicated software - the fMRU plugin [12] for ImageJ [24]. By setting a region of interest (ROI) in the renal parenchyma, the signal is measured over time, creating the signal intensity-time curve (Figure 3). The results were then compared with the information from the diuretic renal scintigraphy.

\section{Diffusion Tensor Imaging}

This technique gives a measure of the anisotropy of diffusion of water molecules. That is the property of the water molecules in a specific structure to diffuse preferentially along predetermined pathways. The architecture of the kidney is particular: in the cortex the glomeruli and tubules are chaotically arranged, therefore water molecules are moving in every direction; in the medulla the tubules are organized in a radial distribution, so the water molecules are moving only in certain directions - it is anisotropic. The anisotropy degree can be quantified and is expressed as the fractional anisotropy (FA) coefficient [25]. The diffusion directions can be visualized by tractography which renders the diffusion vectors (Figure 4). The sequence we used is a modified DTI sequenced used to study the brain. For the kidney we applied the diffusion gradient in six directions $[13,26]$ and used the maximum $b$ value of 400 [27].

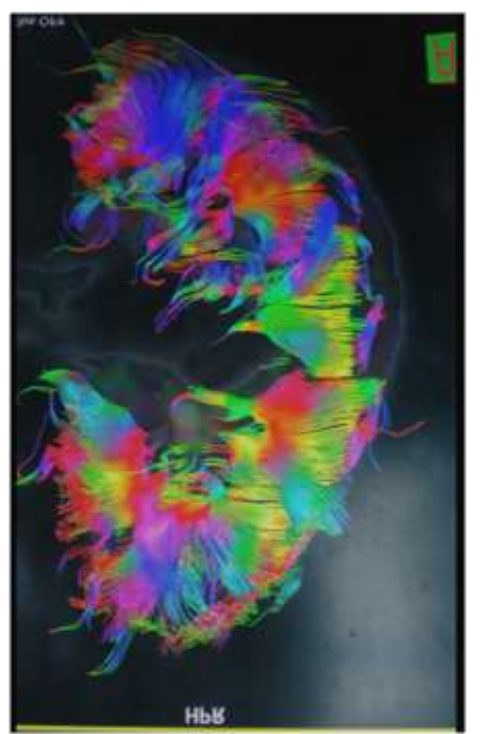

Figure 4 - Tractography in normal kidney. Diffusion directions are color coded: red - left to right; green - head to feet; blue - anterior to posterior (Personal collection).

The scanning is performed in free breathing and takes 60 seconds, but for optimum quality it may be performed with respiratory triggering. The trace, ADC and FA maps are automated generated (Figure 5). We 
imaged 10 patients with no history of renal disease, with the objective of detecting differences in the FA coefficient of medulla and cortex. We then examined 5 patients with obstructed urinary tract to explore if the cortical/medullary FA difference is noted.

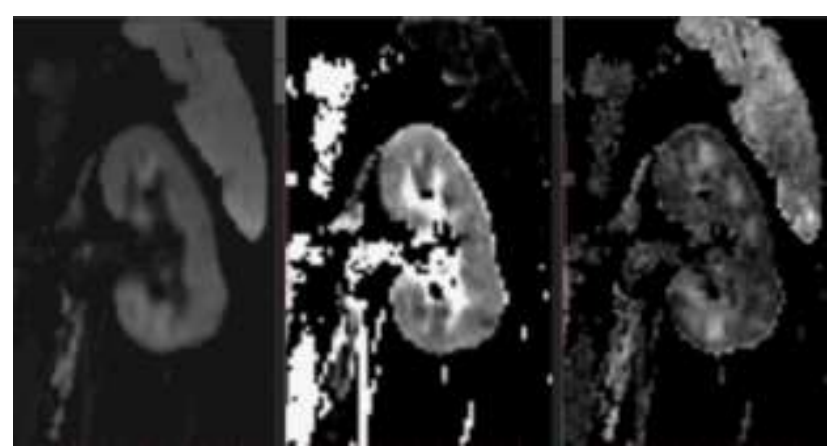

Figure 5 - Trace (left), ADC (middle) and FA map (right) images from DTI. Note the corticomedullary differentiation in the FA map image (Personal collection).

Urodynamic assessment by phase contrast imaging $(P C)$

This method is based on the MRI's high sensitivity to motion. This technique encodes the velocity of moving protons by phase shifts. By measuring those phase shifts, the velocity of those protons can be found. The technique has been developed mainly for cardiovascular studies. We adapted such technique, by altering its parameters making it sensitive to the slow flow of the urine in the collecting system. First, we tested the sequence on 10 normal subjects with no renal disease, and then compared them to one obstructive patient. We performed the measurement of a single slice planned strictly perpendicular to the renal pelvis, collecting data about the flow that goes through the plane. Each measurement takes 2 seconds, so we performed several continuous measurements for 60 seconds. After the acquisition of images, regions of interest were drawn around the contour of the renal pelvis and in a control stationary area and flow parameters were measured - peak velocity, average velocity and flow.

\section{RESULTS}

\section{DCE MR Urography}

The most important part of the curve is the filtration slope. This represents the actual uptake of the Gadolinium by the renal parenchyma. Based on the assumption of bicompartimental distribution of the contrast medium and using linear regression, RutlandPatlak plots are created considering aortic signal as reference (Figure 6). The slope values of the Rutland-Patlak plots for each kidney are compared and thus, the Split Renal Function is determined. To gain more accuracy, these values are combined with the volumetric assessment of each kidney (Figure 7).

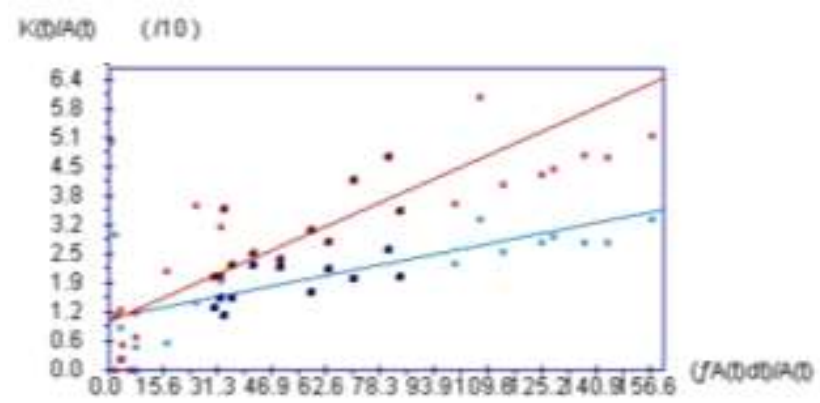

Figure 6 - Rutland-Patlak plots - red: normal kidney; blue: hypofunctional kidney.

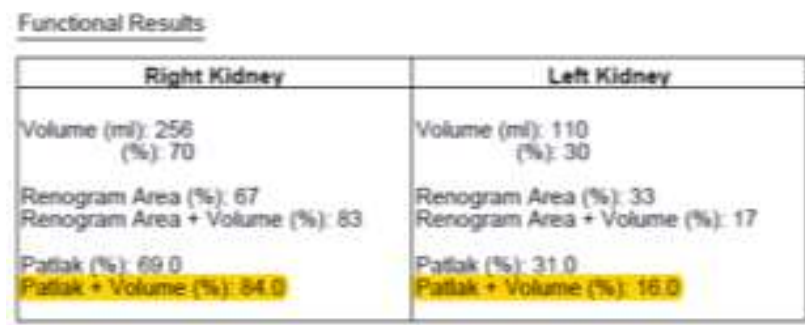

Figure 7 - Calculation output describing the split renal function.

The results observed were similar to the information revealed by renal scintigraphy (Figure 8).

Furthermore, by analyzing the late part of the curve, the excretion curve, a pattern of obstruction is observed in the obstructed kidney (Figure 9). 


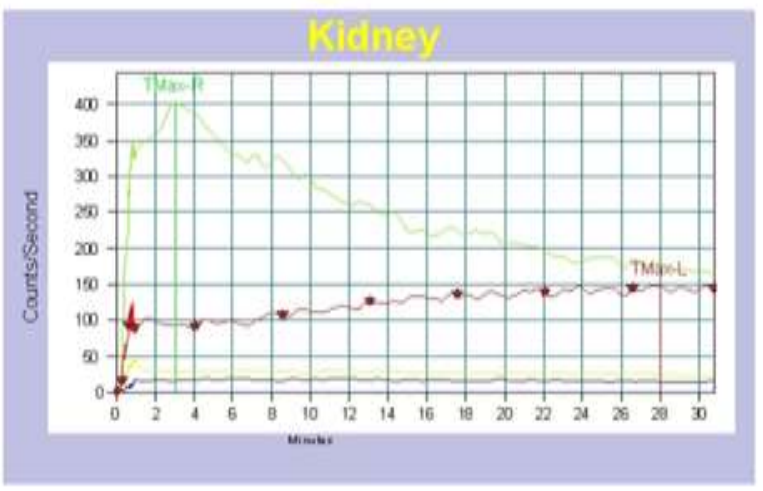

\begin{tabular}{llll}
\hline Table of Result Summary & & & \\
Parameters & Left & Right & Total \\
\hline Split Function (\%) & 20.4 & 79.6 & \\
Kidney Counts (cpm) & 11848 & 46277 & 58125 \\
Kidney Depth (cm) & 4.710 & 4.741 & \\
Uptake (\%) & 1.863 & 7.275 & 9.137 \\
GFR (ml/min) & 16.9 & 66.0 & 82.8 \\
Normalized GFR (ml/min) & & & 98.0 \\
GFR Low Normal (ml/min) & & & 90.0 \\
Mean GFR (ml/min) & & & 118.0 \\
Time of Max (min) & 28.0 & 3.002 & \\
& & & \\
& & & \\
\hline
\end{tabular}

Figure 8 - Renal scintigraphy results. Notice similar split renal function.
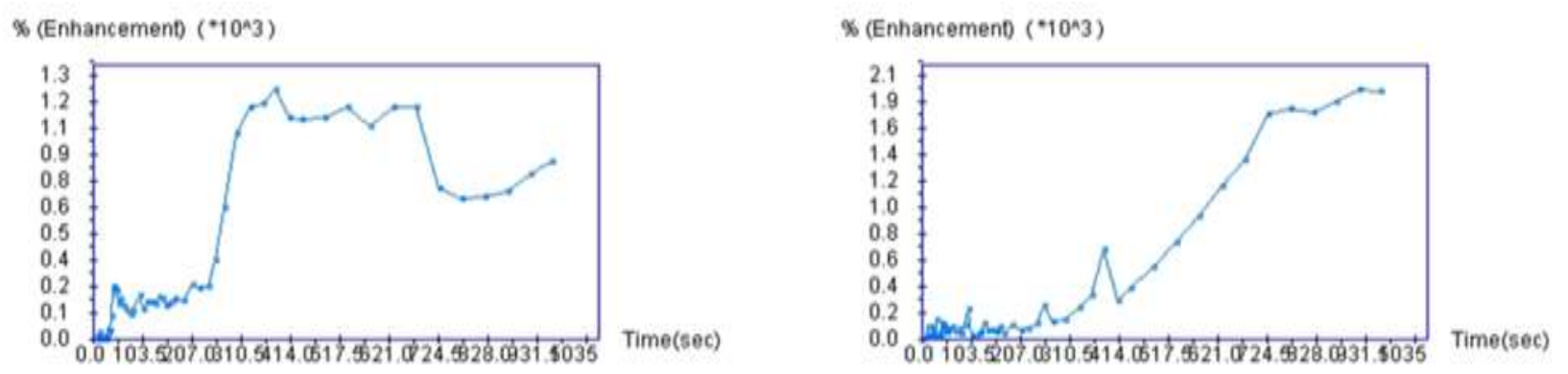

Figure 9 - Excretion curves: left - normal; right - obstruction.

Diffusion Tensor Imaging

In normal kidneys, FA map images reveal a very good cortico-medullary differentiation - the cortex has homogenous low signal, whereas the pyramids are hyperintense (Figure 10).

When measured, the FA values in cortex were significantly lower than in the medulla 0.19 vs. 0.5 [28].

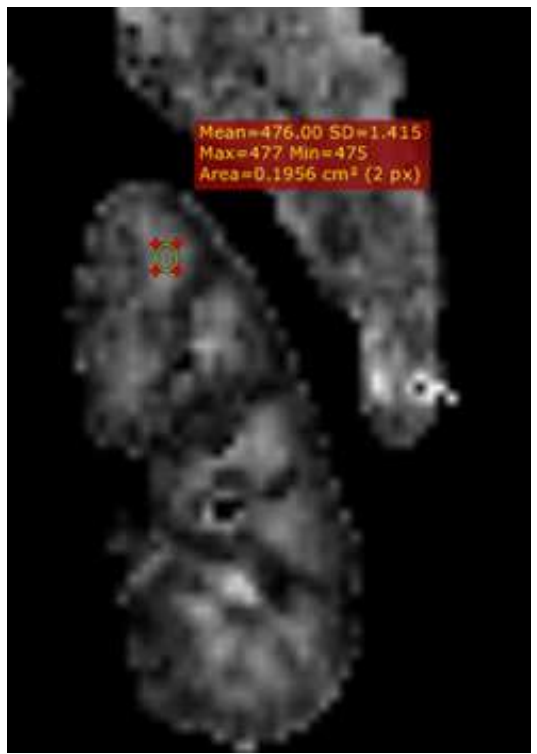

Figure 10 - FA map with measuring the FA coefficient in an upper pyramid.
Tractography reveals tightly packed diffusion tracts in the pyramids, oriented radially towards the papilla (Figure 11).

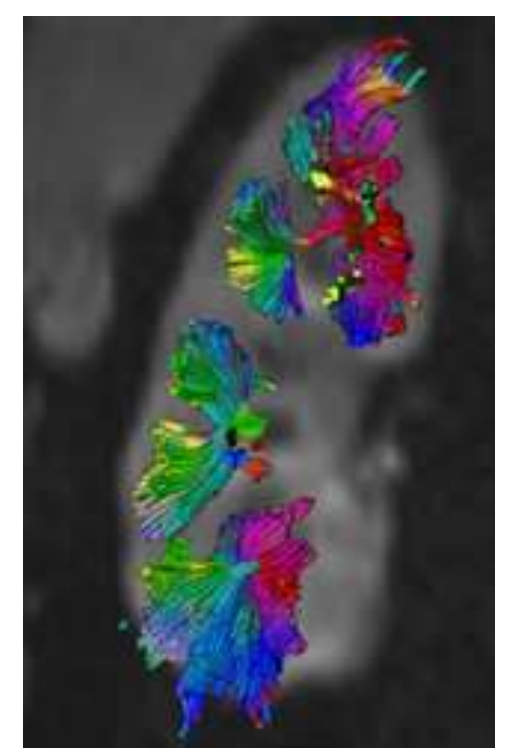

Figure 11 - Tractography image in normal kidney.

In obstructed kidneys with altered renal function, the FA values in medulla decrease considerably, as low as the cortical values (median of 0.15 in cortex and 0.17 in medulla). Tractography reveals changes as well; the medullary tracts in the pyramids are fewer and 
disorganized compared to the normal kidney (Figure 12).
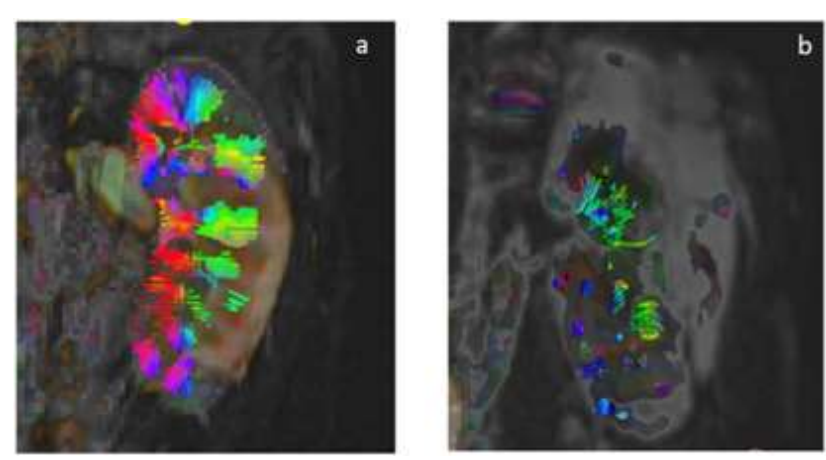

Figure 12 - Tractography images of both kidneys of a patient with Obstruction of the ureteropelvic junction: a - normal kidney; b obstructed kidney.

Urodynamic assessment by phase contrast imaging $(P C)$

In normal kidneys, the urinary flow in the renal pelvis has two components - a baseline low velocity flow over which several peaks of higher velocity are superimposed that correspond to peristaltic waves (Figure 13).

The baseline flow was measured 0.047 $\mathrm{ml} / \mathrm{sec}$, whereas the peristaltic waves were an average of 3 per minute, with velocities up to $1,7 \mathrm{~cm} / \mathrm{s}$.

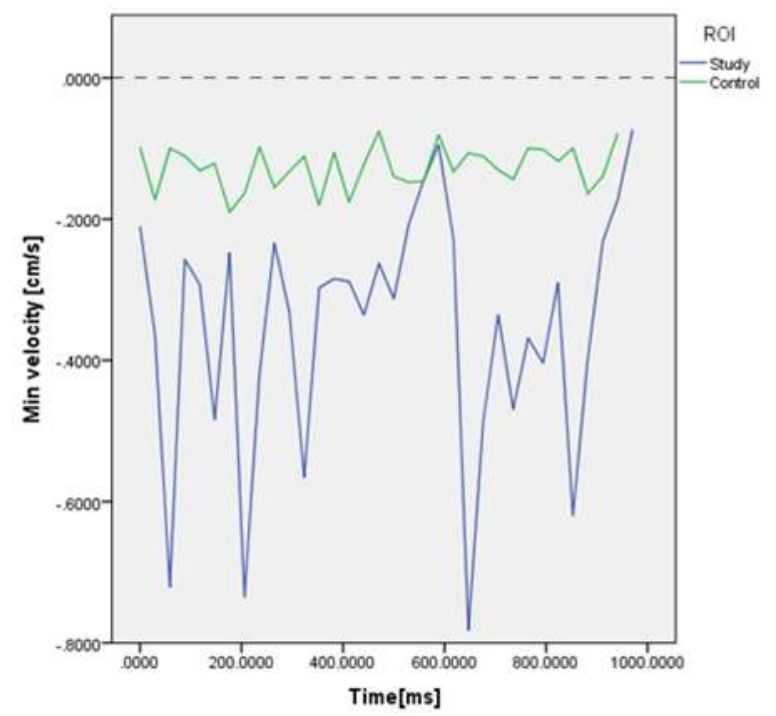

Figure 13 - Peak velocity graph in a normal renal pelvis (blue) compared to control stationary tissue (green).

Next, we assessed the obstructed kidney, and, although we couldn't measure the transstenotic velocity and pressure gradient because of poor resolution, we found fewer peaks of lower amplitude, suggesting poor contractility of long-standing obstructed renal pelvises (Figure 14).

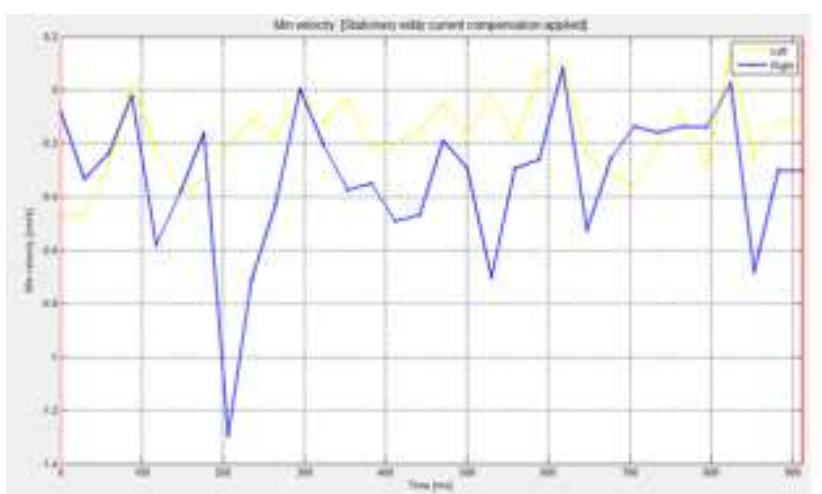

Figure 14 - Peak velocity graph showing a normal pattern of flow in the healthy kidney (blue) and an abnormal flow of the obstructed kidney - few and low amplitude peaks.

\section{DISCUSSIONS}

MRI is famous for delivering exquisite anatomical information about the reno-urinary system. Recent research is oriented towards developing MRI methods of functional imaging of the kidney. Several biomarkers have been studied, each exploring a different aspect of the renal function. Describing the uptake and excretion of Gadolinium based contrast media, DCE-MRI gives information about the glomerular filtration [29,30]. The results are compared for both kidneys, and thus the Split Renal Function is determined. For a more thorough evaluation, volumetric data about the parenchyma is added. This method has been proven accurate compared to conventional renal scintigraphy [31] and several applications were studied. In patients with renal artery stenosis with reno-vascular hypertension, by measuring the GFR by DCE before and after administration of Angiotensin converting enzyme inhibitor, a decrease in GFR was noticed compared to controls without stenosis [6]. In renal transplant, DCE-MRI can distinguish between acute tubular necrosis (ATN) and acute reject by perfusion parameters [32].

In obstructive uropathies, the main role for DCE-MRI is to describe to functional impact of the obstruction. The Split renal Function is calculated, which, in turn is used to guide the treatment. Furthermore, the contrast medium 
clearance from the excretory system can be analyzed to detect obstruction, a feature useful in operated patients with postoperative dilatation.

There are some limitations, however. This technique is based on several assumptions. Firstly, unlike scintigraphy it doesn't measure directly the concentration of the contrast medium, it measures the signal resulted from the magnetic interaction between the contrast medium and the local environment. Thus, the Gadolinium concentration is an estimate based on the magnetic properties of renal tissue. And studies have shown that in vivo values may be different than in vitro ones [33]. Secondly, in another assumption, the contrast medium is distributed only in the vascular and tubular compartments

(bicompartimental pharmacokinetic model), ignoring the possible diffusion outside the tubules [34].

Diffusion Tensor Imaging has the advantage of being an exam without contrast media, thus eliminating concerns about chronic kidney disease patients, or about Gadolinium tissue deposition, especially in children [35]. This technique evaluates damage in the renal tubules. Feasible in a variety of pathologies like chronic kidney disease [36], acute renal injury [37], diabetes [38], polycystic renal disease [18] or renal masses [39], it has been proven useful for congenital urinary obstruction also [40][41]. Jaimes et al [41], for example, detected a significant difference in the FA coefficient in the medulla of obstructed kidneys and medulla of normal kidneys. Thus, kidney DTI provides a quantifiable parameter about the microstructural damage in the renal pyramids. One current limitation is the lack of standardization of the measuring technique between different vendors and different machines.

Urodynamic assessment by phase contrast imaging is a novel technique that we introduced, and although it lacks enough large scales studies, it has potential of delivering of another parameter of a different aspect of renal function. Currently, the gold standard for upper urinary tract urodynamics is the invasive Whitaker test [42]. A tool that would describe the urinary flow non-invasively would be invaluable, especially for dilated systems with non-certain obstruction as is the case for operated patients. The technique we suggest might be able to fill this gap. However more research is needed.

\section{CONCLUSIONS}

In conclusion, renal functional imaging by MRI is an attractive topic as there are several techniques proven to be feasible and other worthy of research. fMRU is also attractive because it studies different aspects of renal function thus helping to better understand different disease processes, non-invasively, without the need for repeating biopsies. However, all these methods have not been standardized for out of the box clinical practice and need to be optimized for each hardware and software setting. Joint effort of medical doctors, medical physicists, engineers and hardware and software vendors is needed to make these techniques reproducible on most machines.

\section{REFERENCES}

[1]Chevalier RL. Congenital Urinary Tract Obstruction: The Long View. Adv Chronic Kidney Dis [Internet]. $2015 \mathrm{Jul} ; 22(4): 312-9$.

[2]Garne E, Loane M, Wellesley D, Barisic I, EUROCAT Working Group. Congenital hydronephrosis: Prenatal diagnosis and epidemiology in Europe. J Pediatr Urol [Internet]. $2009 \mathrm{Feb} ; 5(1): 47-52$.

[3]Lee RS, Cendron M, Kinnamon DD, Nguyen HT. Antenatal Hydronephrosis as a Predictor of Postnatal Outcome: A Meta-analysis. Pediatrics [Internet]. 2006 Aug 1;118(2):586-93.

[4]Riccabona M, Avni FE, Blickman JG, Dacher J$\mathrm{N}$, Darge $\mathrm{K}$, Lobo ML, et al. Imaging recommendations in paediatric uroradiology: minutes of the ESPR workgroup session on urinary tract infection, fetal hydronephrosis, urinary tract ultrasonography and voiding cystourethrography, Barcelona, Spain, June 2007. Pediatr Radiol [Internet]. 2008 Feb 11;38(2):138-45.

[5]Ulman I, Jayanthi VR, Koff SA. The long-term followup of newborns with severe unilateral hydronephrosis initially treated nonoperatively. J Urol [Internet]. 2000 Sep;164(3 Pt 2):1101-5.

[6]Grenier N, Trillaud H, Combe $C$, Degrèze $P$, Jeandot R, Gosse P, et al. Diagnosis of renovascular hypertension: feasibility of captopril-sensitized dynamic MR imaging and comparison with captopril scintigraphy. Am J Roentgenol [Internet]. 1996 Apr;166(4):835-43.

[7]Michaely HJ, Schoenberg SO, Oesingmann N, Ittrich C, Buhlig C, Friedrich D, et al. Renal Artery 
Stenosis: Functional Assessment with Dynamic MR Perfusion Measurements-Feasibility Study. Radiology [Internet]. 2006 Feb;238(2):586-96.

[8]Szolar DH, Preidler K, Ebner F, Kammerhuber F, Horn S, Ratschek $M$, et al. Functional magnetic resonance imaging of human renal allografts during the post-transplant period: preliminary observations. Magn Reson Imaging [Internet]. 1997;15(7):727-35. [9]McDaniel BB, Jones RA, Scherz H, Kirsch AJ, Little SB, Grattan-Smith JD. Dynamic ContrastEnhanced MR Urography in the Evaluation of Pediatric Hydronephrosis: Part 2, Anatomic and Functional Assessment of Uteropelvic Junction Obstruction. Am J Roentgenol [Internet]. 2005 Dec 23;185(6):1608-14.

[10]Rohrschneider WK, Haufe S, Wiesel M, Tönshoff B, Wunsch R, Darge K, et al. Functional and Morphologic Evaluation of Congenital Urinary Tract Dilatation by Using Combined Static-Dynamic MR Urography: Findings in Kidneys with a Single Collecting System. Radiology [Internet]. 2002 Sep 1;224(3):683-94.

[11]Grattan-Smith JD, Little SB, Jones RA. MR urography evaluation of obstructive uropathy. Pediatr Radiol [Internet]. 2008 Jan 11;38(S1):49-69. [12]Vivier P-H, Dolores M, Taylor M, Dacher J-N. MR urography in children. Part 2: how to use ImageJ MR urography processing software. Pediatr Radiol [Internet]. 2010 May 25;40(5):739-46.

[13]Özkan MB, Marterer R, Tscheuner S, Yildirim $\mathrm{UM}$, Ozkan E. The role of kidney diffusion tensor magnetic resonance imaging in children. Egypt $\mathrm{J}$ Radiol Nucl Med [Internet]. 2016 Dec 1;47(4):1599-611.

[14]Jaimes C, Darge K, Khrichenko D, Carson RH, Berman JI. Diffusion tensor imaging and tractography of the kidney in children: feasibility and preliminary experience. Pediatr Radiol [Internet]. 2014 Jan 11;44(1):30-41.

[15]Wang W, Pui MH, Guo Y, Wang L, Wang H, Liu M. 3T magnetic resonance diffusion tensor imaging in chronic kidney disease. Abdom Imaging [Internet]. 2014 Aug 13;39(4):770-5.

[16]Tewes S, Gueler F, Chen R, Gutberlet M, Jang M-S, Meier M, et al. Functional MRI for characterization of renal perfusion impairment and edema formation due to acute kidney injury in different mouse strains. Long D, editor. PLoS One [Internet]. 2017 Mar 20;12(3):e0173248.

[17]Razek AAKA, Al-Adlany MAAA, Alhadidy AM, Atwa MA, Abdou NEA. Diffusion tensor imaging of the renal cortex in diabetic patients: correlation with urinary and serum biomarkers. Abdom Radiol [Internet]. 2017 May 2;42(5):1493500.

[18]Lupica R, Mormina E, Lacquaniti A, Trimboli $\mathrm{D}$, Bianchimano $\mathrm{B}$, Marino $\mathrm{S}$, et al. 3 Tesla-
Diffusion Tensor Imaging in Autosomal Dominant Polycystic Kidney Disease: The Nephrologist's Point of View. Nephron [Internet]. 2016;134(2):7380.

[19]Feng Q, Ma Z, Zhang S, Wu J. Usefulness of diffusion tensor imaging for the differentiation between low-fat angiomyolipoma and clear cell carcinoma of the kidney. Springerplus [Internet]. 2016;5:12.

[20]Artz NS, Sadowski EA, Wentland AL, Grist TM, Seo S, Djamali A, et al. Arterial spin labeling MRI for assessment of perfusion in native and transplanted kidneys. Magn Reson Imaging [Internet]. 2011 Jan;29(1):74-82.

[21]Detre JA, Zhang W, Roberts DA, Silva AC, Williams DS, Grandis DJ, et al. Tissue specific perfusion imaging using arterial spin labeling. NMR Biomed [Internet]. 1994 Mar;7(1-2):75-82.

[22]Li L-P, Vu AT, Li BSY, Dunkle E, Prasad P V. Evaluation of intrarenal oxygenation by BOLD MRI at 3.0T. J Magn Reson Imaging [Internet]. 2004 Nov;20(5):901-4.

[23]Prasad P V, Epstein FH. Changes in renal medullary pO2 during water diuresis as evaluated by blood oxygenation level-dependent magnetic resonance imaging: effects of aging and cyclooxygenase inhibition. Kidney Int [Internet]. 1999 Jan;55(1):294-8.

[24]Schneider CA, Rasband WS, Eliceiri KW. NIH Image to ImageJ: 25 years of image analysis. Nat Methods 2012 97. 2012 Jun 28;

[25]Ries M, Jones RA, Basseau F, Moonen CT, Grenier N. Diffusion tensor MRI of the human kidney. J Magn Reson Imaging [Internet]. 2001 Jul;14(1):42-9.

[26]Palmucci S, Cappello G, Attinà G, Foti PV, Siverino ROA, Roccasalva F, et al. Diffusion weighted imaging and diffusion tensor imaging in the evaluation of transplanted kidneys. Eur J Radiol open [Internet]. 2015;2:71-80.

[27]Chuck NC, Steidle G, Blume I, Fischer MA, Nanz D, Boss A. Diffusion Tensor Imaging of the Kidneys: Influence of $b$-Value and Number of Encoding Directions on Image Quality and Diffusion Tensor Parameters. J Clin Imaging Sci [Internet]. 2013;3:53.

[28]Nicolaescu R, Lupescu I, Preda E, Capsa R, Gherghina I. Diffusion Tensor Imaging-A New Biomarker for Evaluation of Renal Function: Preliminary Results and Literature Review. Rom J Urol [Internet]. 2017;16(1):9-14.

[29]Zhang JL, Rusinek H, Chandarana H, Lee VS. Functional MRI of the kidneys. J Magn Reson Imaging [Internet]. 2013 Feb;37(2):282-93.

[30]Zhang JL, Rusinek H, Bokacheva L, Chen Q, Storey P, Lee VS. Use of cardiac output to improve measurement of input function in quantitative 
dynamic contrast-enhanced MRI. J Magn Reson Imaging [Internet]. 2009 Sep;30(3):656-65.

[31]Bokacheva L, Rusinek H, Zhang JL, Lee VS. Assessment of renal function with dynamic contrastenhanced MR imaging. Magn Reson Imaging Clin N Am [Internet]. 2008 Nov;16(4):597-611, viii.

[32]Szolar DH, Preidler K, Ebner F, Kammerhuber F, Horn S, Ratschek M, et al. Functional magnetic resonance imaging of human renal allografts during the post-transplant period: preliminary observations. Magn Reson Imaging [Internet]. 1997;15(7):727-35. [33]Mørkenborg J, Taagehøj JF, Vaever PN, Frøkiaer J, Djurhuus JC, Stødkilde-Jørgensen H. In vivo measurement of $\mathrm{T} 1$ and $\mathrm{T} 2$ relaxivity in the kidney cortex of the pig--based on a twocompartment steady-state model. Magn Reson Imaging [Internet]. 1998 Oct;16(8):933-42.

[34]Bokacheva L, Rusinek H, Chen Q, Oesingmann N, Prince C, Kaur M, et al. Quantitative determination of Gd-DTPA concentration inT1weighted MR renography studies. Magn Reson Med [Internet]. 2007 Jun;57(6):1012-8.

[35]Guo BJ, Yang ZL, Zhang LJ. Gadolinium Deposition in Brain: Current Scientific Evidence and Future Perspectives. Front Mol Neurosci [Internet]. 2018;11:335.

[36]Wang W, Pui MH, Guo Y, Wang L, Wang H, Liu M. 3T magnetic resonance diffusion tensor imaging in chronic kidney disease. Abdom Imaging [Internet]. 2014 Aug 13;39(4):770-5.
[37]Tewes S, Gueler F, Chen R, Gutberlet M, Jang M-S, Meier M, et al. Functional MRI for characterization of renal perfusion impairment and edema formation due to acute kidney injury in different mouse strains. Long D, editor. PLoS One [Internet]. 2017 Mar 20;12(3):e0173248.

[38]Razek AAKA, Al-Adlany MAAA, Alhadidy AM, Atwa MA, Abdou NEA. Diffusion tensor imaging of the renal cortex in diabetic patients: correlation with urinary and serum biomarkers. Abdom Radiol [Internet]. 2017 May 2;42(5):1493500.

[39]Feng Q, Ma Z, Zhang S, Wu J. Usefulness of diffusion tensor imaging for the differentiation between low-fat angiomyolipoma and clear cell carcinoma of the kidney. Springerplus [Internet]. 2016;5:12.

[40]Özkan MB, Marterer R, Tscheuner S, Yildirim UM, Ozkan E. The role of kidney diffusion tensor magnetic resonance imaging in children. Egypt $\mathbf{J}$ Radiol Nucl Med [Internet]. 2016 Dec;47(4):1599611.

[41]Jaimes C, Darge K, Khrichenko D, Carson RH, Berman JI. Diffusion tensor imaging and tractography of the kidney in children: feasibility and preliminary experience. Pediatr Radiol [Internet]. 2014 Jan 11;44(1):30-41.

[42]Tchetgen M-B, Bloom DA. Robert H. Whitaker and the Whitaker test: a pressure-flow study of the upper urinary tract. Urology [Internet]. 2003 Jan 1;61(1):253-6. 